\title{
SINDICALISMO Y CRISIS ECONÓMICA: AMENAZAS, RETOS Y OPORTUNIDADES
}

\author{
Oriol Barranco \\ Centre d'Estudis Sociològics sobre la Vida Quotidiana i el \\ Treball (QUIT) - Institut d'Estudis del Treball (IET) - Universitat \\ Autònoma de Barcelona

\section{Óscar Molina} \\ Centre d'Estudis Sociològics sobre la Vida Quotidiana i el \\ Treball (QUIT) - Institut d'Estudis del Treball (IET) - Universitat \\ Autònoma de Barcelona
}

RESUMEN: El artículo analiza el impacto que la actual crisis económica está teniendo en los sindicatos españoles y los desafíos, amenazas y oportunidades que ésta les plantea. Se señalan las implicaciones ambivalentes que tienen para los sindicatos los impactos de la crisis en el mercado de trabajo, estado del bienestar, gobernanza y representación política. Se defiende que el deterioro del mercado de trabajo genera un descontento social potencialmente canalizable por los sindicatos, pero también intensifica y agrava las dificultades sindicales para representar a desempleados y trabajadores atípicos. La reducción de la protección y cobertura del estado del bienestar también contribuye a dicho descontento social, pero puede disminuir la confianza ciudadana en la eficiencia de la acción sindical. La crisis de gobernanza pone en cuestión el papel sociopolítico jugado por los sindicatos mayoritarios y les deja sin una de sus fuentes tradicionales de legitimación que era el diálogo social. Finalmente, la crisis del sistema de representación política afecta a los sindicatos disminuyendo la confianza social en ellos. En este contexto, el desafío de los sindicatos es conseguir adaptar su acción a las nuevas circunstancias para que los trabajadores los continúen viendo como útiles y eficientes para defender sus intereses.

Palabras claVe: sindicatos, crisis económica, representación, neocorporatismo, gobernanza. 
RESUM: L'article analitza l'impacte que l'actual crisi econòmica està tenint en els sindicats espanyols i els desafiaments, amenaces i oportunitats que aquesta els planteja. S'assenyalen les implicacions ambivalents que tenen per als sindicats els impactes de la crisi en el mercat de treball, estat del benestar, governança i representació política. Es defensa que el deteriorament del mercat de treball genera un descontentament social potencialment canalitzable pels sindicats, però també intensifica i agreuja les dificultats sindicals per a representar els desempleats i treballadors atípics. La reducció de la protecció i cobertura de l'estat del benestar també contribueix al dit descontentament social, però pot disminuir la confiança ciutadana en l'eficiència de l'acció sindical. La crisi de governança posa en dubte el paper sociopolític que tenen els sindicats majoritaris i els deixa sense una de les seues fonts tradicionals de legitimació que era el diàleg social. Finalment, la crisi del sistema de representació política afecta els sindicats fent que minve la confiança social en aquests. En aquest context, el desafiament dels sindicats és aconseguir adaptar la seua acció a les noves circumstàncies perquè els treballadors els continuen veient com a útils i eficients per a defensar els seus interessos.

Paraules clau: sindicats, crisi econòmica, representació, neocorporatisme, governança.

ABSTRACT: The article analyses the impact the current economic crisis is having on Spanish trade unions and the challenges, threats and opportunities it poses for them. We highlight the ambivalent implications for the unions of the effects the crisis is having on the labour market, the welfare state, governance and political representation. We argue that the deterioration of the labour market generates social unrest that, potentially, the unions can channel, but that it also intensifies and aggravates the difficulties facing trade unions in representing the unemployed and non-typical workers. Reduced protection and coverage from the welfare state also contributes to this social unrest, but it can lower public confidence in the efficiency of trade union action. The crisis of governance calls into question the social and political role played by the main trade unions and leaves them without one of their traditional sources of legitimacy, namely social dialogue. Finally, the crisis of the system of political representation affects trade unions by weakening social trust in them. In this context, the challenge facing the unions is to adapt their actions to the 
new circumstances so workers continue to regard them as useful and efficient defenders of their interests.

KEYWORDS: trade unions, economic crisis, representation, neocorporatism, governance.

\section{Introducción}

$\mathrm{H}^{\mathrm{s}}$

ablar de «crisis» de los sindicatos en el mundo occidental en general, y de los Estados de la Unión Europea en particular, es un tema recurrente desde inicios de los años 70 que ha dado lugar a una abundante literatura especializada, centrada mayoritariamente en el descenso de afiliación (Gallie et al., 1996; Ebbinghaus y Visser, 2000; Visser, 2006). La crisis sindical, sin embargo, es un fenómeno complejo (Ferner y Hyman, 1998) que, además del descenso de la afiliación, incluye, como señala Antentas (2008), las dificultades de representación de determinados colectivos, la reducción de la conflictividad laboral y la reducción de la influencia social de los sindicatos.

Sin embargo, aunque los sindicatos europeos se encuentren inmersos en una situación de crisis desde inicios de los años 70, la crisis financiera internacional de 2008 y la de la deuda soberana en 2010 han supuesto una intensificación de algunas tendencias ya existentes, así como la aparición de nuevas amenazas. De hecho, en algunos países los patrones históricos de acción de los sindicatos han sido en parte desacreditados, obligando a éstos a tomar decisiones estratégicas importantes de cara al futuro (McGumbrell-Cormick y Hyman, 2013). No obstante, tal y como nos recuerda la literatura sobre la revitalización de los sindicatos desde finales de los años 80 (Frege y Kelly, 2003), las crisis no sólo conllevan la necesidad de buscar nuevas estrategias ante las amenazas que puedan plantear, sino que evidencian claramente los principales problemas en la acción sindical y, por tanto, ofrecen al mismo tiempo oportunidades para la re-orientación de algunas de sus políticas. El 
que la percepción social de la necesidad de los sindicatos se mantenga alta en general y no haya dado muestras de declive en la mayoría de los países de la UE (Turner y D'Art, 2012) supone un buen punto de partida para replantear su acción.

El objetivo del presente artículo es analizar el impacto que está teniendo la actual crisis económica en los sindicatos del Estado español y las amenazas, desafíos y oportunidades que ésta les plantea, algunos de los cuáles ya existían y la crisis los ha intensificado mientras otros han emergido con ésta. El artículo se centra fundamentalmente en los dos sindicatos mayoritarios (CCOO y UGT), que son los hegemónicos a nivel estatal desde 1978 y los que en este nivel han participado en el diálogo social tripartito, aunque muchas de las reflexiones realizadas valen también para el resto de sindicatos.

El artículo se organiza en tres secciones. En la primera se analizan las características principales del sindicalismo en el Estado español, para a continuación establecer algunas consideraciones teóricas que ayuden a entender su acción y las consecuencias que la crisis económica está teniendo en la misma. La segunda sección analiza algunos aspectos de la crisis y discute su impacto para los sindicatos. La discusión se centra en los efectos institucionales y en el mercado de trabajo, por una parte, y en la crisis de representación y legitimidad, por otra. Finalmente, la tercera sección contiene algunas consideraciones finales de cara a las políticas que podrían desarrollar los sindicatos.

\section{Sindicatos en el Estado español: retos y estrategias}

El análisis del impacto de la actual crisis económica en los sindicatos españoles ha de tener en cuenta las características institucionales y socioeconómicas específicas del caso español, junto con las tendencias estructurales comunes a los sindicatos occidentales. En este sentido, a continuación se presentan algunos condicionantes estructurales y organizativos que permiten entender la acción de los sindicatos españoles y las consecuencias que la crisis económica está teniendo en la misma. 


\section{I.I. Mercado de trabajo, relaciones industriales y sindicatos}

El primer elemento a considerar son los condicionantes estructurales a los que se enfrentan los sindicatos del Estado español. Algunos de éstos son comunes a otros Estados del entorno, mientras que otros constituyen rasgos distintivos del caso español. Entre los primeros podríamos destacar la creciente precarización y flexibilidad en el mercado de trabajo, provocada por la extensión de nuevas formas de organización empresarial y el rápido crecimiento del empleo en sectores de servicios que requieren por lo general bajos niveles de cualificación. La extensión de formas atípicas de empleo junto al aumento del desempleo, constituye seguramente el principal desafío para los sindicatos desde inicios de los años 80, dadas las dificultades que éstos tienen para atraer y representar a un colectivo cada vez más numeroso y heterogéneo. ${ }^{1}$ Dada su importancia cuantitativa, en el caso español es particularmente importante la extensión del empleo temporal. En principio, la crisis ha supuesto un agravamiento cíclico de estas tendencias, sobre todo en lo que se refiere al aumento del desempleo entre la población más joven e inmigrantes. Sin embargo, se observan algunas dinámicas que, de consolidarse, apuntan a cambios importantes. En particular, el rápido aumento del empleo a tiempo parcial y del número de trabajadore ${ }^{2}$ autónomos plantea nuevos retos para la acción del sindicato en el mercado de trabajo.

El marco institucional en el que desarrolla su acción el sindicato es otro de los elementos que condiciona su posición y acción. A grandes rasgos, podríamos resumir que el caso español se caracteriza por una relativa institucionalización de su acción tanto a nivel general/político (macro), como a nivel de empresa (micro) (Lope y Alós, 1999). A nivel micro, la acción del sindicato se enmarca dentro de las instituciones de representación establecidas en la ley, y que ha consolidado un sistema general de representación de los trabajadores en la empresa que está dominado por los dos sindicatos mayoritarios, ${ }^{3}$ aunque

1. Véase Gumbrell-McCormick (2011) para el desafío que el empleo atípico o precario supone para el sindicalismo a nivel europeo y Miguélez (2004) para el reto que conlleva este tipo de empleo y el desempleo para los sindicatos del Estado español.

2. En el texto se usa el masculino genérico para hacer referencia conjuntamente a hombres y mujeres.

3. Desde 1986 cCOO y UGT acumulan entre el $70 \%$ y $80 \%$ de los delegados electos. 
con algunas excepciones tanto a nivel regional como sectorial. A pesar de esto, la presencia efectiva de los sindicatos en la empresa es relativamente baja comparada con otros países, debido en buena medida a las características del tejido productivo, donde predomina la pequeña y muy pequeña empresa. ${ }^{4}$ La extensión automática de los convenios y acuerdos colectivos dentro del ámbito funcional de éstos (principio erga omnes) garantiza la protección de todos los trabajadores, pero sin embargo ha supuesto una limitación a la extensión de la afiliación entre capas más amplias de trabajadores.

La posición de los sindicatos como actores políticos se fundamenta en un criterio de representatividad que tiene en cuenta los resultados de las elecciones de representantes de los trabajadores y no su afiliación. Los sindicatos «más representativos» (aquéllos que superan el 10\% de delegados electos a nivel estatal) son los que pueden participar en el diálogo social, lo que a nivel estatal significa que sólo lo han podido hacer CCOO y UGT. Así, el diálogo social, a pesar de haber sufrido algunos altibajos, ha sido una de las señas de identidad y una fuente de legitimación de estos dos sindicatos desde la Transición (Molina y Miguélez, 2013).

\section{I.2. Sindicatos y acción política}

A finales de los años 90, en el contexto de ajuste de las economías europeas a los requerimientos impuestos para acceder a la Unión Económica y Monetaria, diversos autores resaltaron el papel que había jugado la acción política de los sindicatos dentro de pactos sociales y de concertación social (Hancke y Rhodes, 2005). La interpretación generalizada que se hizo de estos pactos implicaba una cierta resurrección de prácticas neocorporatistas, a pesar de que no se dieran los requerimientos institucionales establecidos previamente en la literatura (Schmitter, 1974). La vitalidad del diálogo social en España puede observarse en el gran número de acuerdos alcanzados hasta el año 2006 (Molina y Miguélez, 2013). El debate sobre la revitalización sindical tomó estos pactos y el creciente papel político desempeñado por las

4. Según datos de la ECTV 2010 recogidos por Beneyto (2010), la cobertura de la representación sindical es del $82 \%$ en las empresas de más de 250 trabajadores, del $49 \%$ en las de 10 a 49 y del $25 \%$ en las de 1 a 9 . 
organizaciones sindicales como una de las estrategias sobre las cuales debía cimentarse su renovación social y organizativa. Ésta debía acompañarse, sin embargo, de acciones complementarias encaminadas a consolidar su papel a nivel micro, es decir, a consolidar su capacidad de representación y defensa de los intereses de los trabajadores a nivel de empresa (Frege y Kelly, 2003).

Sin embargo, tal y como señalaba la literatura sobre neocorporatismo (Streeck, 1988), la acción política del sindicato implica una verticalización organizativa, que puede entrar en conflicto con otra de las señas distintivas del movimiento sindical: la participación de los miembros en la organización. En otras palabras, el neocorporatismo (entendido tanto como una forma de organización y representación de intereses como una forma de elaborar políticas) contenía una deriva centralizadora que era difícilmente compatible con la democracia interna. Como consecuencia de esto, las organizaciones participantes en estructuras y procesos neocorporatistas tenían altas probabilidades de experimentar problemas de representación a medio y largo plazo.

En este mismo sentido, Colin Crouch hacía referencia a la necesidad de articulación de las organizaciones sindicales y patronales para que un sistema neocorporatista de relaciones laborales se consolidase (Crouch, 1993; Molina, 2008). De acuerdo con su argumento, sólo en aquellos países donde tanto sindicatos como empresarios hubiesen desarrollado estructuras fuertes tanto a nivel micro (empresa) como a nivel macro, y donde existiesen mecanismos eficaces de comunicación y participación, sería posible consolidar un sistema neocorporatista de relaciones laborales. Según esta visión, una organización con escasa implantación a nivel de empresa, pero que jugase un papel político importante y donde no existiesen mecanismos de participación de los diferentes niveles en las decisiones de la cúpula, sería difícilmente sostenible.

\section{Las consecuencias de la crisis económica para el sindicalismo}

La profundidad, larga duración y gestión política de la crisis económica iniciada en 2008 ha provocado la emergencia de una crisis en el mercado de trabajo, una crisis social, así como una crisis de gobernanza y representación políticas. A partir del análisis de los condicionantes tanto estructurales como 
organizativos comentados en los párrafos anteriores, la siguiente sección analiza cuál ha sido el impacto de la crisis en sus múltiples manifestaciones, y el impacto de éstas en los sindicatos.

\section{I. La crisis del mercado de trabajo y sus consecuencias}

La crisis económica ha comportado un deterioro significativo del mercado de trabajo, incrementando y agudizando el problema del desempleo masivo y empeorando las condiciones de una buena parte de los empleos existentes. El desempleo ha crecido fuertemente, alcanzando su récord histórico en el primer trimestre de 2013 (6,2 millones y una tasa del 27,16\%, EPA) y estando a finales de 2013 en 5,9 millones de personas y en una tasa del $26 \%$, que en los menores de 25 se eleva al 55\%. El sector público ha contribuido notablemente a este aumento como consecuencia de los recortes (Recio, 2013). Se han incrementado también las situaciones de paro más duras y difíciles: el $60 \%$ de los parados llevan más de un año en esa situación, el 40\% no recibe ninguna prestación y 1,83 millones de hogares tienen a todos sus miembros en paro. ${ }^{5}$

Por otro lado, a pesar de la importante destrucción de empleos temporales que ha supuesto la crisis, a finales de 2013, según la EPA, casi el 24\% del empleo continuaba siendo de este tipo, y se incrementan dentro de éstos los contratos de menos de un mes de duración (del 25\% en 2005 al 38\% a finales de 2013). Al mismo tiempo, han aumentado los contratos a tiempo parcial (del $25 \%$ en 2005 al $35 \%$ a finales de 2013), ${ }^{6}$ que en España son mayoritariamente involuntarios. Globalmente, los salarios descienden, tanto en el sector público como en el privado, y pierden poder adquisitivo: en un 10,8\% de media y en un $18,1 \%$ entre los obreros no cualificados entre 2007 y 2011 (Martínez-García, 2014). ${ }^{7}$ Así, aumenta el porcentaje de «trabajadores pobres» (del 23\% en 2007 al 28\% en 2012) ${ }^{8}$ y el autoempleo como una opción de subsistencia frente al desempleo (Recio, 2013).

5. Datos de la epa del cuarto trimestre de 2013.

6. Para un análisis en profundidad, ver Carrasquer (2013).

7. Calculados a partir de los datos de las Encuestas de Condiciones de Vida de 2008 y 2012 (ine).

8. Ver http://elpais.com/elpais/2014/01/24/opinion/1390564773_396809.html 
Las respuestas gubernamentales a la crisis del mercado de trabajo han pasado por diversas etapas. Después de una primera de negación de la crisis económica e inacción ante la misma por parte del gobierno del PSOE, en otoño de 2008 se responde con el primer rescate bancario, la financiación de obra pública y la aprobación en 2009 de la ayuda no contributiva de 426 euros de 6 meses para los desempleados sin derecho a prestación contributiva. Sin embargo, con el estallido de la crisis de la deuda soberana en 2010, el mismo gobierno socialista aplicará medidas de austeridad acorde con las propuestas de la troika, que en política de empleo tienen como objetivo reducir al máximo el gasto público en empleo (tiempo y nivel de prestación) y hacer reformas que reduzcan el coste del trabajo y/o faciliten el despido (Miguélez, 2013 a). La Reforma Laboral del 2010, aprobada sin el acuerdo de patronal ni sindicatos, abarató el despido, amplió las causas para llevar a cabo despidos colectivos, potenció la flexibilidad numérica interna y abrió la posibilidad de que las empresas pudieran aplicar cláusulas de convenios de empresa que vulneraran convenios de ámbito superior.

Posteriormente, con el gobierno del Partido Popular en el poder desde noviembre de 2011, se profundiza y radicaliza el proceso de reforma del mercado de trabajo en 2012. Esta reforma permite que el empleador unilateralmente cambie las condiciones laborales (incluyendo el salario o la jornada), y se modifica la denominada ultra-actividad de los convenios colectivos estableciendo que de no llegarse a un acuerdo el marco regulatorio sea un convenio de ámbito superior, y de no existir, el Estatuto de los Trabajadores. Además, la reforma también reduce la capacidad reguladora de los convenios regionales y sectoriales frente a los de empresa, un nivel en el que muchos trabajadores tienen un débil poder de negociación, pues muchos están empleados en pequeñas empresas de menos de 50 asalariados con poca o nula presencia sindical (el 47,5\%). ${ }^{9}$ Por último, el Real Decreto-ley 16/2013, de 20 de diciembre, permite que el empresario unilateralmente cambie la organización del trabajo. Con estas reformas se facilita que los empresarios puedan cambiar las condiciones laborales de sus empleados y, como señala Baylos (2012), deprecia el rol negociador y regulador de los sindicatos, así como su poder negociador.

9. Dato del Ministerio de Empleo y Seguridad Social 2012. 
El empeoramiento de la situación laboral de una gran mayoría de trabajadores, junto a la incertidumbre y pesimismo sobre cuándo puede producirse una mejora, han provocado un elevado malestar, preocupación y descontento de una mayoría de la población con la situación del empleo y del mercado de trabajo. Así parece desprenderse del barómetro del CIS, que muestra cómo, desde mediados de 2008, entre el 75 y el $80 \%$ de los encuestados consideran el paro como uno de los tres principales problemas del país y hasta principios de 2014 entre el 40 y el 50\% consideran así los problemas económicos; además, en octubre de 2014, a pesar del discurso gubernamental sobre la llegada de la recuperación económica, el 70,3\% creía que la situación económica en un año sería igual o peor.

Las consecuencias de este deterioro del mercado de trabajo y del empleo para la acción sindical son de alto calado y de distinto tipo, generando tanto potencialidades como dificultades. Del lado de las potencialidades, el descontento con la situación del empleo y del mercado de trabajo, de acuerdo a lo señalado por distintos autores de la acción colectiva (por ejemplo, Gurr, 1970; Gamson, 1992; Klandermans, 1994), podría ser fuente inicial o primaria de acción y organización colectiva. La situación, potencialmente, debería poder favorecer que una parte de esas personas descontentas decidieran organizarse y/o participar en acciones colectivas para cambiar el estado de cosas que genera el descontento. El reto sindical es atraer a esas personas, o a parte de ellas, a los sindicatos y sus acciones, en forma de afiliación y/o de participación en los actos de protesta, sea en las empresas o en el territorio.

Sin embargo, el deterioro del mercado de trabajo también incrementa la intensidad de algunas dificultades sindicales existentes previamente. En primer lugar, el hecho de que la mayoría de personas se vinculen a los sindicatos en los centros de trabajo y se desvinculen de los mismos cuando pierden el empleo, ha provocado una disminución de la afiliación sindical como efecto del incremento del desempleo. En este sentido, Beneyto (2011) muestra un descenso de la afiliación del 5,9\% entre 2007 y $2010 .{ }^{10}$ Sin embargo, al tratarse de un descenso inferior al sufrido en el mismo período por la población ocupada $(-10,1 \%)$ y la asalariada $(9,2 \%)$, de acuerdo con Beneyto, mostraría

10. Beneyto basa su cálculo en los datos en la Encuesta de Condiciones de Vida y Trabajo. 
una notable capacidad de resistencia sindical a la crisis. En segundo lugar, el alto desempleo y los muchos ERE de los años de crisis es de suponer que pueden haber aumentado la sensación de vulnerabilidad y de miedo a la pérdida del empleo en muchos trabajadores, lo que podría actuar como desincentivo para la afiliación y la participación en las acciones sindicales por el miedo de muchos de ellos a ser mal vistos por su empleador y que ello pudiera ir en su contra. En tercer lugar, el incremento de contratos inferiores a un mes de duración implica el aumento de trabajadores con una situación contractual débil y vulnerable y que suele limitar el compromiso en las luchas laborales en las empresas. Además, estos contratos dificultan enormemente que los trabajadores afectados puedan integrarse en las plantillas y desarrollar en éstas los vínculos estables y fuertes que suelen requerirse para el trabajo y la acción sindical. Por último, el aumento de trabajadores autónomos incrementa la importancia del problema sindical de cómo llegar a este colectivo.

\subsection{La crisis social y sus consecuencias}

Las políticas de austeridad fiscal han conllevado una importante reducción del gasto público social y un incremento de la recaudación mediante la subida del impuesto sobre la renta, de impuestos indirectos como el IVA y de las tasas de algunos servicios públicos.

La reducción del gasto social ha implicado una reducción de la protección social y la bajada de la calidad y cobertura de diversas prestaciones y servicios públicos, en un Estado del Bienestar que ya presentaba importantes déficits e insuficiencias de cobertura en diversas áreas (Guillén y León, 2011). El Estado del Bienestar que se fue construyendo en las tres décadas precedentes al estallido de las crisis económica se fundamentaba en un sistema educativo, un sistema sanitario y unas pensiones contributivas, considerados sus tres pilares, con buena cobertura e intensidad protectoras (González, 2008), pero con el resto de servicios y prestaciones presentando importantes carencias y déficits que los hacían incapaces de cubrir a toda la población que los requería, ni con la intensidad que los necesitaba (Rodríguez Cabrero, 2004). Éste era el caso de las políticas públicas de vivienda, los servicios de guardería, centros de día y atención a las personas dependientes, etc. 
La reducción presupuestaria de las distintas administraciones públicas sumadas (central, autonómica y local), según Muñoz de Bustillo y Antón (2013), podría cifrase, desde 2008 hasta 2013, alrededor del 15\% en educación y sanidad, mientras para el resto de partidas sociales sería de un 20\%. Este ajuste presupuestario ha supuesto la reducción de personal y la rebaja salarial en las administraciones públicas, así como importantes modificaciones en muchas prestaciones y servicios públicos: eliminación de la ayuda para el alquiler a los jóvenes de 22 a 29 años introducida en 2007, endurecimiento de los requisitos para recibir la renta mínima de inserción, progresiva paralización de la ayuda a la dependencia y reducción de la cuantía percibida en la prestación por desempleo a partir del sexto mes (la tasa de reposición se disminuye del $60 \%$ al 50\%). Pero, además, los sistemas educativo y sanitario y las pensiones, los tres pilares del estado de bienestar español, sufren importantes modificaciones ${ }^{11}$ que dificultan, reducen y/o pueden impedir el acceso a los mismos de algunas personas.

Las consecuencias sociales de todo ello son importantes. En primer lugar, el $60-65 \%$ de ciudadanos ${ }^{12}$ que se calcula que cubren sus necesidades de reproducción social fundamentalmente mediante el sistema público están viendo rebajadas la cobertura e intensidad protectora que les proporcionaban las prestaciones y servicios públicos. Se trata, evidentemente, de una afectación desigual en función de la renta y las necesidades de los usuarios. En segundo lugar, se ha incrementado el riesgo de dualización en el acceso a la protección social y a los servicios educativos y sanitarios entre los colectivos sociales más vulnerables y necesitados de asistencia social y aquéllos con más recursos que pueden acceder a los mismos en el mercado (Molina y Godino, 2013). En tercer lugar, la renta familiar disponible y su poder adquisitivo se han reducido: según datos del INE, en 2012 la renta media de los españoles (18.500 euros) era inferior en términos de capacidad adquisitiva a la que existía en 2001 (Fundación Foessa, 2013). En cuarto lugar, se ha incrementado la desigualdad en la distribución de la renta: desde el 2007 al 2012, el índice de Gini ha aumentado casi un $10 \%$ y la diferencia entre la renta de los percentiles 80 y 20 ha crecido un $30 \%$. Y, por último, una proporción creciente

11. Una lista detallada puede encontrarse en Muñoz de Bustillo y Antón (2013).

12. Cálculo aportado por Navarro (2006). 
de población está llegando a niveles muy altos de vulnerabilidad (Fundación Foessa, 2013).

Las consecuencias para la acción sindical vuelven a ser ambivalentes, existiendo potencialidades pero también riesgos. La potencialidad es que el malestar y descontento ciudadano existente con la situación y los gobernantes puede ser una base para atraer más voluntades hacia la acción sindical. Ha de tenerse en cuenta que una buena parte de los ciudadanos afectados y descontentos son base real (afiliados o participantes en actos sindicales) y otra parte son base potencial de la acción sindical, pues son población trabajadora en diversas situaciones laborales (empleados, parados, e incluso trabajadores autónomos). El reto para los sindicatos es conseguir ser vistos por más ciudadanos como unas instituciones y actores sociopolíticos que defienden sus intereses sociales y los protegen de manera efectiva.

El riesgo es que la situación provoque una disminución de la confianza y/o la legitimidad de los sindicatos frente a los ciudadanos que forman su base social, potencial y real. Esto puede ocurrir de dos modos. En primer lugar, existe un riesgo de que algunos ciudadanos dejen de creer o confiar en la eficacia de los sindicatos para defender sus intereses al percibir que durante los años de la crisis éstos han sido incapaces de defender los intereses de los trabajadores en las políticas gubernamentales. En segundo lugar, otros ciudadanos incluso podrían dejar de confiar en los sindicatos por considerarlos corresponsables de la situación actual ${ }^{13}$ por su acción política articulada a través de la concertación y el diálogo social.

\subsection{Crisis de gobernanza y sus consecuencias}

El modo y los ámbitos en que se han venido decidiendo las políticas de austeridad han afectado a la gobernanza democrática (Molina y Miguélez, 2013). La crisis ha tenido el efecto de concentrar y centralizar el poder político en las élites económicas y políticas supranacionales (Jessop, 2013) y, como había predicho Crouch (2009), ha profundizado en los procedimientos postdemocráticos en la toma de decisiones económicas, como ha sucedido

13. Incluso una parte del $15 \mathrm{M}$ o de los Indignados compartía esta visión sobre los sindicatos, que eran considerados parte del sistema que había que cambiar. 
con los memorandos y paquetes europeos, con la instauración en su momento de gobiernos técnicos en Grecia e Italia, así como en el hecho de que las líneas maestras de políticas económicas y sociales de facto se estén tomando en instancias superiores a los parlamentos nacionales, concretamente la troika y el gobierno Alemán (Miguélez, 2013 a).

La crisis de gobernanza tiene implicaciones fundamentales para la concertación social tripartita entre Gobierno, sindicatos y patronal. Por un lado, porque los actores socioeconómicos nacionales ven reducida su capacidad efectiva de influir en la situación socioeconómica. Por otro lado, porque la nueva forma de gobernanza, según Pastor (2013), intenta sustituir dicha concertación por una política del «shock» que busca reducir al máximo el poder asociativo y de negociación de los asalariados. En el caso español, Molina y Miguélez (2013) muestran que al comienzo de la crisis el Gobierno del PSOE mantuvo el diálogo social tripartito en diversas cuestiones pero sin conseguir la firma de ningún acuerdo relevante. Sin embargo, el estallido de la crisis de la deuda en 2010 dinamitó la concertación social. El Gobierno del PSOE cambia de orientación y, aunque formalmente mantuvo el diálogo social tripartito, toma decisiones unilateralmente, como la reforma laboral de $2010 \mathrm{y}$ el primer paquete de medidas de austeridad, a las que se oponen los sindicatos. Con la llegada al poder en 2011 del gobierno del PP, éste adopta una aproximación unilateral de toma de decisiones, ignora el diálogo social tripartito (Gago, 2012; Molina y Miguélez, 2013) y profundiza en las políticas de austeridad. De este modo, la concertación tripartita no ha tenido ningún rol significativo en la política económica ni fiscal desde el comienzo de la crisis (Molina y Miguélez, 2013) y los sindicatos ven erosionada su posición de actor político (Köhler et al., 2012).

Los sindicatos mayoritarios se encuentran, pues, frente a un nuevo escenario político en el que se les niega y cuestiona el papel de actor político que habían tenido. Frente a ello, los dos sindicatos mayoritarios hacen un giro hacia una mayor contestación. Como muestran Köhler et al. (2012), en el período 2008-2012, los sindicatos han convocado tres huelgas generales, han solicitado la convocatoria de un referéndum sobre las políticas de austeridad y han convocado, directamente o al lado de otras organizaciones, diversas concentraciones y manifestaciones contra las políticas gubernamentales. Pro- 
testando contra las políticas de austeridad los sindicatos se han encontrado con otros actores sociales, como el 15M durante un tiempo o las «mareas ciudadanas» en defensa de la sanidad y la educación públicas. De momento, sin embargo, ni los sindicatos, ni los otros movimientos sociales, ni su acción conjunta han conseguido parar las medidas de austeridad.

La nueva situación tiene implicaciones importantes para la acción sindical. En primer lugar, la inutilidad y posterior ruptura de la concertación social tripartita supone para los sindicatos mayoritarios quedarse sin un tipo de acción de la que obtenían legitimidad y credibilidad frente a una parte de su base social. El reto es que los sindicatos mantengan o incrementen su legitimidad mediante nuevas fuentes, como por ejemplo su rol de organizadores de movilización social contra las políticas de austeridad y las transformaciones del modelo social que está teniendo lugar. En segundo lugar, el hecho de que las decisiones claves respecto a las políticas económicas, sociales y laborales se estén dictando más allá de las instituciones de gobernanza nacionales, concretamente por la troika, así como la dimensión transnacional que tiene la crisis y las políticas de austeridad, vuelve a poner sobre la mesa el problema de la necesaria organización internacional de las luchas y protestas sociales en general y de las laborales en particular. Las resistencias sindicales a las políticas de austeridad se están produciendo fundamentalmente a escala nacional, a pesar de los intentos de sobrepasarla con las convocatorias del CES (Confederación Europea de Sindicatos), de los Días Europeos de la Acción y la Solidaridad contra la Austeridad. En tercer lugar, el hecho de que los sindicatos no sean los únicos actores sociopolíticos que protestan contra las políticas de austeridad y que nadie sea suficientemente fuerte para cambiar la orientación de las políticas socioeconómicas plantea a los sindicatos la cuestión de configurar alianzas con los otros movimientos sociales que luchan contra las actuales políticas de gestión de la crisis.

\subsection{Crisis de representación política y sus consecuencias}

Por último, existe también una crisis de confianza y una pérdida de legitimidad del sistema político de representación de intereses y de una buena parte del sistema institucional. La desafección y baja confianza en el sistema político y en varias de sus instituciones es una tendencia que viene de lejos, 
pero durante los años de crisis económica se ha profundizado e intensificado. Medina (2013), a partir de los datos de la Encuesta de Opinión del ICPS, Instituto de Ciencias Políticas y Sociales, muestra que mientras en 2007 el $37 \%$ de los encuestados expresaba su insatisfacción con el funcionamiento del sistema político, en el 2012 lo eran el 76\%. Con datos de otra encuesta, Tezanos y Díaz (2013) muestran que un 77,5\% de encuestados considera que en el Estado español existen problemas de representación política que requieren reformas.

El descontento con el sistema de representación política también toma la forma de un descenso a mínimos históricos de la valoración y confianza ciudadanas hacia sus instituciones y actores centrales, tanto públicas como privadas. Según diversas encuestas, esto les sucede al sistema judicial, la Iglesia católica, la Monarquía y las distintas administraciones públicas (central, autonómica y local). Las valoraciones y confianzas más bajas (bajísimas) son para el sistema bancario, los partidos políticos y los políticos. Toharia (2013), a partir del Barómetro de confianza institucional de Metroscopia de julio de 2013, muestra que sólo evalúa positivamente a los bancos el 15\%, a los partidos políticos el $12 \%$ y a los políticos un raquítico $6 \%$. Otros datos significativos son que por primera vez desde la recuperación de la democracia la intención directa de abstenerse haya superado a la de votar a cualquier partido $^{14}$ o que para un $27 \%$ de la población los políticos y los partidos formen parte de uno de los tres problemas principales del país (Barómetro CIS, enero 2014). Destaca especialmente la caída de la confianza y la mala valoración ciudadana de los dos partidos mayoritarios que han venido gobernando el Estado los últimos decenios y que han gestionado políticamente la crisis económica mediante las políticas de austeridad. En el barómetro del cis de octubre de 2014, el 68'9 de los encuestados valoran como mala o muy mala la acción del gobierno del PP y el 63,8\% considera así la de la oposición del PSOE. Ambos partidos pierden intención de apoyo electoral y si en las elecciones generales de 2008 ambos conjuntamente acumulaban el 83,81\% de los votos, las estimaciones del cIs de octubre de 2014 apuntan a que ambos acumularían el 51,4\%. Igualmente revelador e indicador del proceso de desgaste de estos

14. Como señalan Mateos y Penadés (2013), sucedió por primera vez en el Barómetro del CIS de julio de 2012. En el barómetro de enero de 2014 continua sucediendo. 
partidos es el rápido y espectacular crecimiento de la intención de voto a Podemos señalada por el barómetro del cIs, que en octubre de 2014 le señalaban como el primer partido en intención directa de voto y con un voto estimado del 22 '5\%. Sin duda, este nuevo partido estaría canalizando y capitalizando una parte del malestar social existente entre la ciudadanía con la situación económica, social y política.

Paralelamente, aumenta la movilización ciudadana en manifestaciones (cuyo número se dispara desde 2008) y otros actos de protesta, que alcanzan los niveles más altos desde la Transición. El interés por la política también aumenta. De acuerdo con Medina (2013), éste ha ido aumentado desde 2007, y en el 2012 los que afirmaban estar muy o bastante interesados en la política eran el $47 \%$, que son a su vez los que más críticos se muestran con las instituciones.

Las razones fundamentales del incremento durante los años de crisis económica de la insatisfacción y descontento ciudadano con el sistema institucional y político de representación de intereses y sus principales actores, probablemente, se encuentran en el efecto combinado de tres factores. Un primer factor es el descontento con las políticas seguidas en la gestión de la crisis (Medina, 2013). Por un lado, porque se considera que estas medidas han implicado graves injusticias en el reparto de los sacrificios económicos (Sánchez-Cuenca, 2014). Por otro, porque la no llegada de la recuperación económica, y que ni se perciba cuándo puede llegar, es probable que esté generando entre muchos ciudadanos la opinión de que las medidas adoptadas eran inútiles y desacertadas. De tenerse esta visión, parece lógico pensar que ésta pueda haber derivado en desconfianza y descontento, primero, en los gobiernos, partidos y políticos que las están aplicando y, segundo, en todas aquellas instituciones y/o actores que puedan parecer corresponsables de dichas políticas. Un segundo factor son los casos de corrupción y fraude, señalados como uno de los tres problemas principales del país por el $42 \%$ de los encuestados en el barómetro del cis de octubre de 2014. Por último, en la merma de la confianza ciudadana en partidos y políticos, parece lógico pensar que el incumplimiento de las promesas y programas electorales está contribuyendo a ello. 
Los sindicatos parecen verse arrastrados también por el descontento ciudadano hacia las instituciones y actores centrales del actual modelo de representación político. Así, por ejemplo, los barómetros del cIs muestran que mientras en 2006 los sindicatos eran puntuados con una media de 4,22 sobre diez, en 2013 lo eran en un 2,45.

La explicación de la bajada de la confianza en los sindicatos, probablemente, puede deberse a dos tipos de visiones. Puede que algunas personas crean que los sindicatos están perdiendo capacidad para defender adecuadamente los intereses de los trabajadores, sea como incapacidad en el nivel de empresa de evitar ERE, bajadas salariales, etc., sea como actor social para evitar las políticas de austeridad. Pero puede ser también que otras personas perciban a los sindicatos como corresponsables de la mala situación social y/o connivencia con algunas políticas gubernamentales, por formar parte del sistema de representación político. En este último caso, el reto sindical es, como propone Miguélez (2013 b) mejorar su capacidad de comunicar y explicar lo que pueden y no pueden hacer en relación al empleo y las políticas gubernamentales.

Por último, y globalmente, el reto sindical que genera esta bajada de confianza está, evidentemente, en subirla, fundamentalmente entre la parte de la ciudadanía que puede considerarse su base social. Para ello, la acción sindical debe mostrar su utilidad y eficiencia en la defensa de los intereses de los trabajadores. El reto está en conseguirlo haciendo frente y adaptando su acción a las nuevas y difíciles circunstancias.

\section{Consideraciones finales}

A partir del análisis de los efectos de la crisis, hemos intentando proveer un panorama en el que entender los retos, amenazas y oportunidades que ésta plantea para los sindicatos y su acción en el caso español. Dos son los elementos más novedosos que ha planteado la crisis económica y que tienen un impacto directo sobre la acción de los sindicatos del Estado español. En primer lugar, la dimensión europea ha tomado un papel hasta ahora desconocido en la gobernanza de las políticas de respuesta y ajuste, y por tanto ha limitado en un doble sentido el papel de los actores nacionales, incluyendo los sindi- 
catos. Primero, porque la eficacia de algunas de las políticas clave en el ajuste dentro de la unión económica y monetaria, como es por ejemplo la salarial, depende en gran parte de las acciones llevadas a cabo en otros Estados miembros. Por lo tanto, uno de los temas sobre los que tradicionalmente ha pivotado el diálogo social y los pactos sociales como mecanismo de ajuste se halla en gran parte determinado desde fuera. Segundo, porque la imposición de las políticas de ajuste por las instituciones de gobierno económico a nivel europeo ha limitado la posibilidad de acción de los actores sociales nacionales.

El segundo aspecto más novedoso que la reciente crisis económica ha supuesto para la acción sindical es la creciente desafección y crítica ciudadanas hacia las instituciones y actores políticos. En particular, existe un descontento creciente acerca de la calidad de las instituciones democráticas y su capacidad para resolver los problemas sociales que se plantean actualmente. Este descontento se extiende también a los sindicatos, sobre todo en su calidad de actores políticos que han hecho de la concertación social uno de sus ejes fundamentales de acción. Los problemas derivados de la centralización organizativa que ha conllevado esta estrategia son, sobre todo, una pérdida de la capacidad legitimadora que una estructura más participativa puede implicar.

Ante esta situación, las líneas de acción estratégica de los sindicatos podrían tener una doble dirección, hacia arriba y hacia abajo. Hacia arriba, es necesario que los sindicatos de los diversos Estados de la UE unan fuerzas e incrementen su coordinación para devenir un actor real a escala europea. Con ello responderían en parte a la crisis de gobernanza que se mencionaba anteriormente. Hacia abajo, es necesario que los sindicatos desarrollen mecanismos que impliquen, por una parte, una mayor participación de las bases $y$, por otra, una mayor imbricación y arraigo a nivel local/regional, donde la relación entre los miembros y la organización es más cercana y por tanto puede contribuir a atenuar la crisis de confianza y legitimidad a que se ha hecho referencia.

\section{Referencias}

Antentas, J. M. (2008): «Los sindicatos ante la globalización. ¿Hacia qué nuevas formas de solidaridad internacional?», Cuadernos de Relaciones Laborales, vol. 26, $\mathrm{n}^{\circ} 1,35-55$. 
BAYlos, A. (2012): «El sentido general de la reforma: ruptura de los equilibrios organizativos y colectivos y la exaltación del poder privado del empresariado», Revista de Derecho Social, n $57,9-18$

Beneyto, P. J. (2010): «Situación actual del sindicalismo. Un análisis sociológico». En Beneyto, P. J. (coord.): Reivindicación del sindicalis$m o$. Fundación $1^{\circ}$ de Mayo, Madrid.

Beneyto, P. J. (2011): «La afiliación sindical durante la crisis». En: <http:// www.fsc.ccoo.es/comunes/recursos/17487/965841-La_afiliacion_ sindical_durante_la_crisis,_por_Pere_J._Beneyto.pdf $>$.

Carrasquer, P. (2013): «¿Viejas fórmulas para nuevas realidades? El empleo a tiempo parcial en el modelo de empleo en España», Ponencia en XI Congreso Español de Sociología, Madrid, 10-12 julio 2013.

Crouch, C. (1993): Industrial Relations and European State Traditions. Oxford University Press, Oxford.

Crough, C. (2009): «Privatised Keynesianism: An Unacknowledged Policy Regime», British Journal of Politics \& International Relations, 11 (3).

Ebbinghaus, B. y J. Visser (1999): «When Institutions Matter: Union Growth and Decline in Western Europe, 1950-1995», European Sociological Review, 15 (2), 1-24.

Ferner, A. y R. Hyman (eds.) (1998): Changing Industrial Relations in Europe, Blackwell, London.

Frege, C. y J. Kelly (2003): «Union Revitalization Strategies in Comparative Perspective», European Journal of Industrial Relations, 9 (1), pp. 7- 24

Fundación Foessa (2013): «Los indicadores sociales». En Análisis y perspectivas 2013. Desigualdad y derechos sociales, Fundación Foessa, Madrid, pp. 4-21.

GAGo, A. (2012): «Los sindicatos mayoritarios españoles, ccoo y UGT, ante la crisis económica: ¿Declive o revitalización?». En AguiLar, S. (ed.): Anuario del conflicto social 2012, Observatori del Conflicte social, pp. 1075-1104.

Gallie, D.; R. Penn y M. Rose (1996): Trade Unionism in Recession, Oxford University Press, Oxford.

Gamson, W. A. (1989): The Strategy of Social Protest, Watsworth, Belmont.

GonzÁlez, J. J. (2008): «Estado de bienestar y desigualdad». En GonzÁlez, J. J. y M. REQuena (eds.): Tres décadas de cambio social en España. ( $2^{\mathrm{a}}$ edición), Alianza Editorial, Madrid. 
Guillén, A. M. y M. León (2011): «Conclusions». En Guillén, A. M. y M. LEÓN (eds.): The Spanish Welfare State in European Context. Ashgate, Farnham.

Gumbrell-McCormick, R. (2011): «European trade unions and "atypical" workers», Industrial Relations Journal, vol. 42 (3): 293-310

Gumbrell-McCormick, R. y R. Hyman (2013): Trade Unions in Western Europe: Hard Times, Hard Choices. Oxford University Press, London.

GuRR, T. (1970): Why men Rebel? Princetown University Press, Princetown.

Hnacke, R. y M. Rhodes (2005): «EMU and Labor Market Institutions in Europe. The Rise and Fall of National Social Pacts», Work and Occupations, 32(2), 196-228

JEssop, R. (2013): «Recovered Imagineries, Imagined Recoveries: A Cultural Political Economy of Crisis Construals and Crisis Management in the North Atlantic Financial Crisis». En Benner, M. (Ed.): Before and Beyond the crisis. Edward Elgar, Cheterlman (UK) -Massachussetts (US).

Klandermans, B. (1984): «Mobilisation and Participation: Social-Psicological Expansions of Resource Mobilisation Theory». American Sociological Review, Vol. 49.

KöHLer, H-D.; S. González y D. Luque (2012): «Sindicatos, crisis económica y repertorios de protesta en el sur de Europa». En Anuario del conflicto social, Observatori del Conflicte Social, 1013-1052.

Lope, A. y R. Alós (1999): «La acción sindical en la empresa. Entre el desconcierto y la reacción». En Miguelez, F. y C. Prieto (coords): Las relaciones de empleo en España, Siglo xxI, Madrid, pp 213-237.

Martínez-García, J. S. (2014): «¿Cómo afecta la crisis a las clases sociales?», Zoom Político, Laboratorio de alternativas.

Mateos, A. y A. Penadés (2013): «España: Crisis y recortes», Revista de Ciencia Política, 1, 161-183.

Medina, L. (2013): «Les institucions en el punt de mira. Les valoracions de les institucions i dels actors polítics i socials entre la població catalana (1991-2012)», Quaderns de l'ICPS. Institut de Ciència Polítiques i Socials, Barcelona.

Miguélez, F. (2004): «Presente y futuro del sindicalismo en España». En BENEYTo, P. J. (comp.): Afiliación sindical en Europa, vol.1, Ediciones Germanía, Valencia.

MiguéLez, F. (2013 a): «¿Están fallando las políticas de empleo?», Anuario IET, vol.1, 143-156. 
Miguélez, F. (2013 b): «La democracia en los sindicatos. La importancia de la democracia participativa en la acción sindical», Gaceta Sindical, 21, 171-182.

Molina, O. (2008): «Social Pacts, Collective Bargaining and Trade Union Articulation Strategies», Transfer: European Journal of Labour and Research, 14(3), 399-418

Molina, O. y A. Godino (2013): «Economic Reforms and the Labor Market: Zapatero's Endless Period in the Wilderness». En FIELD, B. N. y A. Bотті (eds.): Politics and Society in Contemporary Spain: From Zapatero to Rajoy, Palgrave Macmillan, London.

Molina, O. y F. Miguélez (2013): «From Negotiation to Imposition: Social Dialogue in Austerity Times in Spain», Working Paper, 51, International Labour Office, ILO, Geneva.

Muñoz de Bustillo y J-I. Antón (2013): «Those Were the Days, My Friend: The Public Sector and the Economic Crisis in Spain». En VAUGHAN-Whitehead, D. (ed.): Public Sector Shock. The Impact of Policy Retrenchment in Europe. Edward Elgar, Cheterlman (UK) - Massachussetts (US).

Navarro, V. (2006): El subdesarrollo social de España, Anagrama, Barcelona.

Pastor, J. (2003): «Crisis de régimen y refundación (im)possible», Viento Sur, 28.

ReCio, A. (2013): «2012: La segunda recesión», Anuario IET, vol.1, 1-20.

Rodríguez Cabrero, R. (2004): El Estado del bienestar en España: debates, desarrollo y retos, Fundamentos, Madrid.

Sánchez-Cuenca, I. (2014): La impotencia democrática. Sobre la crisis política de España, La Catarata, Madrid.

Schmitter, P. (1974): «Still the Century of Corporatism?», The Review of Politics, 36 (1), 85-131

STreeck, W. (1988): «Editorial Introduction to Special Issue on Organizational Democracy on Trade Unions», Economic and Industrial Democracy, 9, 307-318

Tezanos, J. F. y V. Díaz (2013): «La izquierda se convierte en la fuerza electoral potencialmente mayoritaria en España», Dossier, 23, en: http://www.fundacionsistema.com/media/public/doc/Dossier $\% 20$ 23 T228.pdf

Toharia, J. J. (2013): «La imagen social de los sindicatos. Acotaciones sobre datos de opinión recientes», Gaceta Sindical, 21, 163-170. 
Turner, T. y D. D’Art (2012): «Public Perceptions of Trade Unions in Countries of the European Union: A Causal Analysis», Labor Studies Journal, 37(1), 33-55.

Visser, J. (2006): «Union Membership Statistics in 24 Countries», Monthly Labor Review, enero, 3-14. 
